\title{
Multidimensional membership functions in T-S fuzzy models for modelling and identification of nonlinear multivariable systems using genetic algorithms
}

\author{
José Miguel Adánez Basil Mohammed Al-Hadithi ～Agustín Jiménez
}

\begin{abstract}
A B S T R A C T
In this work, a new method for Takagi-Sugeno ( $T$-S) fuzzy modelling based on multidimensional membership functions (MDMFs) is proposed. It is verified that the fuzzy inference method of one-dimensional membership functions (1DMFs) may place the fuzzy rules in inappropriate locations for modelling of nonlinear multivariable systems, while the application of MDMFs allows a better identification through a smaller number of fuzzy rules. The proposed method uses a genetic algorithm (GA) for the adjustment of the MDMFs and the T-S method for modelling and identification of the nonlinear system. As a validation example, a nonlinear multivariable system, a coupled tanks system, is chosen. The results show that the proposed method presents less identification error than the T-S method, with less number of fuzzy rules.
\end{abstract}

\section{Introduction}

Fuzzy logic [1] has become an important technique for artificial intelligence, since the fuzzy logic allows to introduce the human uncertain behaviour to the computer definite performance. Fuzzy logic has become one of the most popular tools for modelling and control of nonlinear systems, especially the T-S fuzzy model [2], which allows the global identification of a nonlinear system as a set of linearized systems by fuzzy bending. Many works have been developed with T-S fuzzy models [3-5].

In [6], an approach developed by the authors is presented to improve the estimation of $\mathrm{T}-\mathrm{S}$ models. The problem is that the original $\mathrm{T}-\mathrm{S}$ identification method cannot be applied when the triangular membership functions are overlapped by pairs. This restricts the use of this type of membership functions which have been widely used in the controllers design and are popular in industrial applications. The approach, to search for an exact optimal solution, uses the minimum norm method although it increases complexity and computational cost. Another approach was developed in [7], which can be considered as a generalized version of T$\mathrm{S}$ method. This simple method with not much computational cost is based on weighting of parameters. In [8], this T-S identification method was extended to the multivariable case. These methods are characterized by the high accuracy obtained for modelling nonlinear systems in comparison with the original T-S method [2].

One of the main problems of the traditional fuzzy based methods, for multidimensional fuzzy space, is the automatic inference of 1DMFs. The fuzzy inference is an easy method to obtain fuzzy 
rules, but the generated rules can be placed in inappropriate locations, which usually are not optimal and sometimes even do not correspond to the system data.

In order to solve this problem, the MDMFs [9] can be applied. The main idea is to generate the membership functions in the multidimensional space, instead of generate 1DMFs to be combined with fuzzy inference. These MDMFs can be located wherever the designer decides, but some algorithms have been developed to facilitate this task [10]. In [11], a clustering algorithm (fuzzy cmeans [12]) is used for MDMFs location.

There are some variations of fuzzy clustering algorithms which obtain the fuzzy rules for the best separation of data in clustering problems. Some examples of this algorithms are fuzzy Cmeans [13-15], fuzzy possibilistics C-means [16], Gustafson-Kessel [17-19], Gath-Geva [20], and other approaches [21]. Instead of these clustering methods, our approach is based on the MDMFs location using a GA. An important advantage of the GA based proposed method with respect to deterministic clustering fuzzy approaches is that the proposed objective function for the GA is designed to find the MDMFs parameters which minimizes directly the $\mathrm{T}-\mathrm{S}$ identification error.

The GAs $[22,23]$ are optimization methods which use the principles of natural selection and genetics to find the optimal solution to a problem. The main advantage of the GAs is the inclusion of random changes in the solutions, which avoids that the algorithm falls in a local minimum permanently. As a main drawback, the GAs do not ensure the optimal solution in finite time, but with enough number of generations (the iterations of the GA), the GAs usually give good results with a reasonable computational cost. Several works present different applications of the GAs [24-26].

The GAs have been used with fuzzy based methods in some works [27-29]. The most prominent is the genetic fuzzy rule based system whose genetic process adjusts different components of a fuzzy system $[30,31]$. However, in our approach the GAs has been used for finding the best MDMFs location for a T-S identification. Once the MDMFs are successfully designed and the T-S identification is made, it is easy to create a fuzzy controller [32,33], which is out of the scope of this paper.

Summarizing, in this work it is verified that the fuzzy inference of 1DMFs may place the fuzzy rules in inappropriate locations for modelling and control of nonlinear multivariable systems. Thus, in order to solve that, it is proposed the use of MDMFs which allows a better identification through a smaller number of fuzzy rules. A GA is proposed for the MDMFs automatic adjustment since this algorithm allows the convergence of the MDMFs parameters in a reasonable computing time. An illustrative example of a coupled tanks system is presented, which clearly shows the advantages of the proposed method.

The rest of this work is organized as follows. In Section 2, the T-S identification method based on 1DMFs inference is described. In Section 3, the T-S model based on MDMFs is explained. The adjustment method for MDMFs based on GAs is presented in Section 4. In Section 5, an illustrative example of a coupled tanks system is presented to show the advantages of the proposed MDMFs adjusted by a GA over the traditional fuzzy inference of 1DMFs

\section{T-S identification method}

The identification method of T-S fuzzy models is based on estimating the nonlinear system parameters minimizing a quadratic performance index [2]. The traditional T-S identification method fails if the triangular membership functions of the fuzzy rules are overlapped by pairs, since the T-S matrix is not of full rank and thus it is not invertible [7]. Therefore, the authors in [7,8] proposed a generalized T-S identification, using a parameters weighting method.
The method is based on the identification of nonlinear functions which can be modelled as a set of difference equations by the following IF-THEN rules for an $n$th order system of $s$ outputs and $p$ inputs:

$$
\begin{aligned}
\mathrm{S}^{\left(i_{1} \ldots i_{m}\right)} & \text { If } z_{1}(k) \text { is } M_{1}^{i_{1}} \text { and } \ldots \text { and } z_{m}(k) \text { is } M_{m}^{i_{m}} \text { then: } \\
y_{i}(k+1) & =a_{i 0}^{\left(i_{1} \ldots i_{m}\right)}+a_{i 1}^{\left(i_{1} \ldots i_{m}\right)} y_{i}(k)+\cdots+a_{i n_{i}}^{\left(i_{1} \ldots i_{m}\right)} y_{i}\left(k-n_{i}+1\right) \\
& +\sum_{j=1}^{p} b_{i j 1}^{\left(i_{1} \ldots i_{m}\right)} u_{j}(k)+\cdots+b_{i j n_{i}}^{\left(i_{1} \ldots i_{m}\right)} u_{j}\left(k-n_{i}+1\right)
\end{aligned}
$$

where $\left\{y_{1}(k), y_{2}(k), \ldots y_{s}(k)\right\}$ are the system outputs, $\left\{u_{1}(k)\right.$, $\left.u_{2}(k), \ldots u_{p}(k)\right\}$ are the system inputs and $\left\{z_{1}(k), z_{2}(k), \ldots z_{m}(k)\right\}$ are measurable variables, which hereinafter will be called fuzzy variables. In this fuzzy notation, $j$ is the fuzzy variable index and $i_{j}$ is the fuzzy rule index associated with the fuzzy variable. The fuzzy estimation of the output becomes:

$$
\begin{aligned}
& \hat{y}_{i}(k+1)=\sum_{i_{1}=1}^{r_{1}} \ldots \sum_{i_{m}=1}^{r_{m}} \beta^{\left(i_{1} \ldots i_{m}\right)}\left(z_{\left(i_{1} \ldots i_{m}\right)}(k)\right) \\
& \times\left[a_{i 0}^{\left(i_{1} \ldots i_{m}\right)}+a_{i 1}^{\left(i_{1} \ldots i_{m}\right)} y_{i}(k)+\cdots\right. \\
& +a_{i n_{i}}^{\left(i_{1} \ldots i_{m}\right)} y_{i}\left(k-n_{i}+1\right)+\sum_{j=1}^{p} b_{i j 1}^{\left(i_{1} \ldots i_{m}\right)} u_{j}(k)+\cdots \\
& \left.+b_{i j n_{i}}^{\left(i_{1} \ldots i_{m}\right)} u_{j}\left(k-n_{i}+1\right)\right]
\end{aligned}
$$

where

$\beta^{\left(i_{1} \ldots i_{m}\right)}\left(z_{\left(i_{1} \ldots i_{m}\right)}(k)\right)=\frac{\prod_{j=1}^{m}\left(\mu_{j i_{j}}\left(z_{j}(k)\right)\right)}{\sum_{i_{1}=1}^{r_{1}} \ldots \sum_{i_{m}=1}^{r_{m}}\left(\prod_{j=1}^{m}\left(\mu_{j j_{j}}\left(z_{j}(k)\right)\right)\right)}$

with $\mu_{j i}\left(z_{j}\right)$ being the membership function corresponding to the fuzzy set $M_{j}^{i_{j}}$. For the parameters weighting method, supposing that a first affine linear estimation model is available. In other words, a first estimation of the following parameters is known:

$P_{0}=\left[\begin{array}{llllllllll}a_{i 0}^{0} & \ldots & a_{i n_{i}}^{0} & b_{i 11}^{0} & \ldots & b_{i 1 n_{i}}^{0} & \ldots & b_{i p 1}^{0} & \ldots & b_{i p n_{i}}^{0}\end{array}\right]$

In order to obtain this first estimation, the classical least squares method can be applied to the data set of the system. This estimation can be used as reference parameters for all the T-S fuzzy model subsystems

$$
p_{0}=\underbrace{\left[\begin{array}{cccc}
P_{0} & P_{0} & \cdots & P_{0}
\end{array}\right]^{t}}_{r_{1} \cdot r_{2} \cdots r_{m}}
$$

Then, the fuzzy model parameters can be obtained minimizing

$$
\begin{aligned}
J & =\sum_{k=1}^{n_{S}}(y(k)-\hat{y}(k))^{2}+\gamma^{2} \sum_{i_{1}=1}^{r_{1}} \ldots \sum_{i_{m}=1}^{r_{m}} \sum_{j=0}^{n}\left(p_{j}^{0}-p_{j}^{\left(i_{1} \ldots i_{m}\right)}\right)^{2} \\
& =\|Y-X P\|^{2}+\gamma^{2}\left\|p_{0}-P\right\|^{2} \\
& =\left\|\left[\begin{array}{c}
Y \\
\gamma p_{0}
\end{array}\right]-\left[\begin{array}{c}
X \\
\gamma I
\end{array}\right] P\right\|^{2}=\left\|Y_{a}-X_{a} P\right\|^{2}
\end{aligned}
$$

where $Y$ are the output data, $X$ are the fuzzy input/output data and $P$ are the parameters of the fuzzy model.

The $\gamma$ factor represents the degree of confidence of the parameters initially estimated $[7,8]$. Note that the matrix $X_{a}$ is of full rank, even if the triangular membership functions are overlapped by pairs. This solves the problem while the original T-S identification method fails. Thus the vector $P$ can be computed as:

$P=\left(X_{a}^{t} X_{a}\right)^{-1} X_{a}^{t} Y_{a}$ 
In case if the state variables are measurable [8], Eq. (1) can also be represented by a set of discrete state-space models as:

$$
\begin{aligned}
& S^{\left(i_{1} \ldots i_{m}\right)} \text {. If } z_{1}(k) \text { is } M_{1}^{i_{1}} \text { and } \ldots \text { and } z_{m}(k) \text { is } M_{m}^{i_{m}} \text { then: } \\
& x(k+1)=a_{0}^{\left(i_{1} \ldots i_{m}\right)}+A^{\left(i_{1} \ldots i_{m}\right)} x(k)+B^{\left(i_{1} \ldots i_{m}\right)} u(k)
\end{aligned}
$$

Thus, the fuzzy identification can be made with state models [8] using a similar formulation of Eqs. (2) and (3).

Note that, in the T-S identification method, the fuzzy rules weights $w^{\left(i_{1} \ldots i_{m}\right)}=\prod_{j=1}^{m}\left(\mu_{j j_{j}}\left(z_{j}\right)\right)$ are automatically generated by 1DMFs $\mu_{j i_{j}}\left(z_{j}\right)$ inference. The fuzzy rules generated by fuzzy inference, may not be suitable for optimal identification, even though the $1 D M F s$ are well chosen, which is verified by an illustrative example in Section 5. In this work, the MDMFs are proposed to solve this problem.

\section{T-Sfuzzy model identification based on MDMFs}

The 1DMFs fuzzy inference can generate some rules which are not optimal for the fuzzy model, and even do not correspond to the system data, which produces a loss of optimality and an unnecessary computational cost.

MDMFs can be used to improve the model identification. Its main advantage is that they are directly generated in the multidimensional space. Some algorithms are used for automatic adjustment of MDMFs, such as clustering algorithms [9,11]. MDMFs can be designed with different shapes, for example n-dimensional pyramids [10].

Our approach with multidimensional fuzzy sets $M^{i}$ is as follows. The fuzzy variable is defined as:

$z=\left[\begin{array}{llll}z_{1} & z_{2} & \cdots & z_{f}\end{array}\right]^{t}$
$z \in \Re^{f}$

and the MDMF $\mu_{i}(z)$.

For each fuzzy set $M^{i}$, a central point $z_{i} \in \Re^{f}$ is defined and for each $z(k) \in \mathfrak{R}^{f}$, a quadratic distance is defined as:

$$
d_{i}(z(k))=\sum_{j=1}^{f} p_{i j}\left(z_{j}(k)-z_{i j}\right)^{2} \quad p_{i j} \geq 0
$$

where $p_{i} \in \Re^{f}$ is a design parameter vector which weights the distances.

The MDMF (Fig. 1) is:

$\mu_{i}(z(k))=\frac{1}{1+d_{i}(z(k))}$

which has the advantage of being 1 for the central point and between 0 and 1 for the rest of the space. The function decreases as the points move away from the central point. In addition, the function is derivable with respect to any parameter.

This approach can be considered as an approximation of a Gaussian radial-basis function with less computational cost. This is important because the same fuzzy rules designed for modelling and identification stages must also be applied at control stage, which is online and therefore, the computing time becomes crucial.

The T-S fuzzy model of the system with $n_{r}$ rules is:

$$
S^{i}: \text { If } z(k) \text { is } M^{i} \text { then: }
$$

$$
\begin{aligned}
y_{1}(k+1) & =a_{10}^{i}+a_{11}^{i} y_{1}(k)+\cdots+a_{1 n_{1}}^{i} y_{1}\left(k-n_{1}+1\right) \\
& +\sum_{j=1}^{p} b_{1 j 1}^{i} u_{j}(k)+\cdots+b_{1 j n_{1}}^{i} u_{j}\left(k-n_{1}+1\right)
\end{aligned}
$$

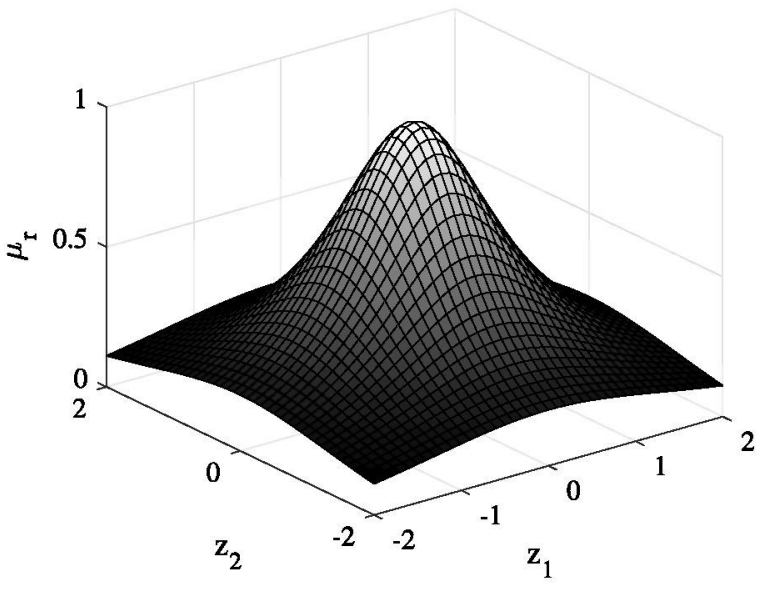

Fig. 1. Proposed MDMF.

And the system becomes:

$$
\begin{aligned}
\hat{y}_{1}(k+1) & =\sum_{i=1}^{n_{T}} \alpha_{i}(z(k))\left[a_{10}^{i}+a_{11}^{i} y_{i}(k)+\cdots\right. \\
& +a_{1 n_{1}}^{i} y_{1}\left(k-n_{1}+1\right) \\
& \left.+\sum_{j=1}^{p} b_{1 j 1}^{i} u_{j}(k)+\cdots+b_{1 j n_{1}}^{i} u_{j}\left(k-n_{1}+1\right)\right]
\end{aligned}
$$

where

$\alpha_{i}(z(k))=\frac{\mu_{i}(z(k))}{\sum_{i=1}^{n_{r}} \mu_{i}(z(k))}$

\section{MDMFs design based on GA}

In the previous section the MDMF functions (7) are defined, but the MDMFs parameters $\left(z_{i}\right.$ and $\left.p_{i}\right)$ must be calculated for an optimal T-S identification. In order to obtain the MDMFs parameters (antecedents) and the T-S model parameters (consequents), several methods applying different adjustment algorithms have been assayed.

The first algorithm is based on the optimal adjustment of MDMFs through a variation of an algorithm developed by the authors in $[34,35]$. The algorithm performs a recursive adjustment of the T-S antecedents through an extended Kalman filter [36]. Unfortunately, with this method, the antecedents convergence is not guaranteed. A second method consists on applying an optimization algorithm based on the gradient descent method [37]. However, this algorithm is too slow and the obtained antecedents can correspond to a local minimum.

Finally, a method based on GAs is applied [22,23]. The T-S antecedents are adjusted by a GA, whereas the T-S consequents are obtained by the T-S identification method [2].

The proposed GA follows the next steps, shown in Fig. 2:

1. An initial population, composed by a set of individuals, is defined. Each individual of the population is formed by a fuzzy system, made by $n_{r}$ fuzzy rules. In each fuzzy rule, a set of antecedents is determined, $\left\{z_{i}\right\} \in \mathfrak{R}^{f}$ and $\left\{p_{i}\right\} \in \mathfrak{R}^{f}$, which defines a MDMF. The initial antecedents of the fuzzy system are specified for each individual with different values. Thus, firstly, the number of fuzzy rules $n_{r}$, the number of individuals, and a set of initials MDMFs for each individual; should be determined 


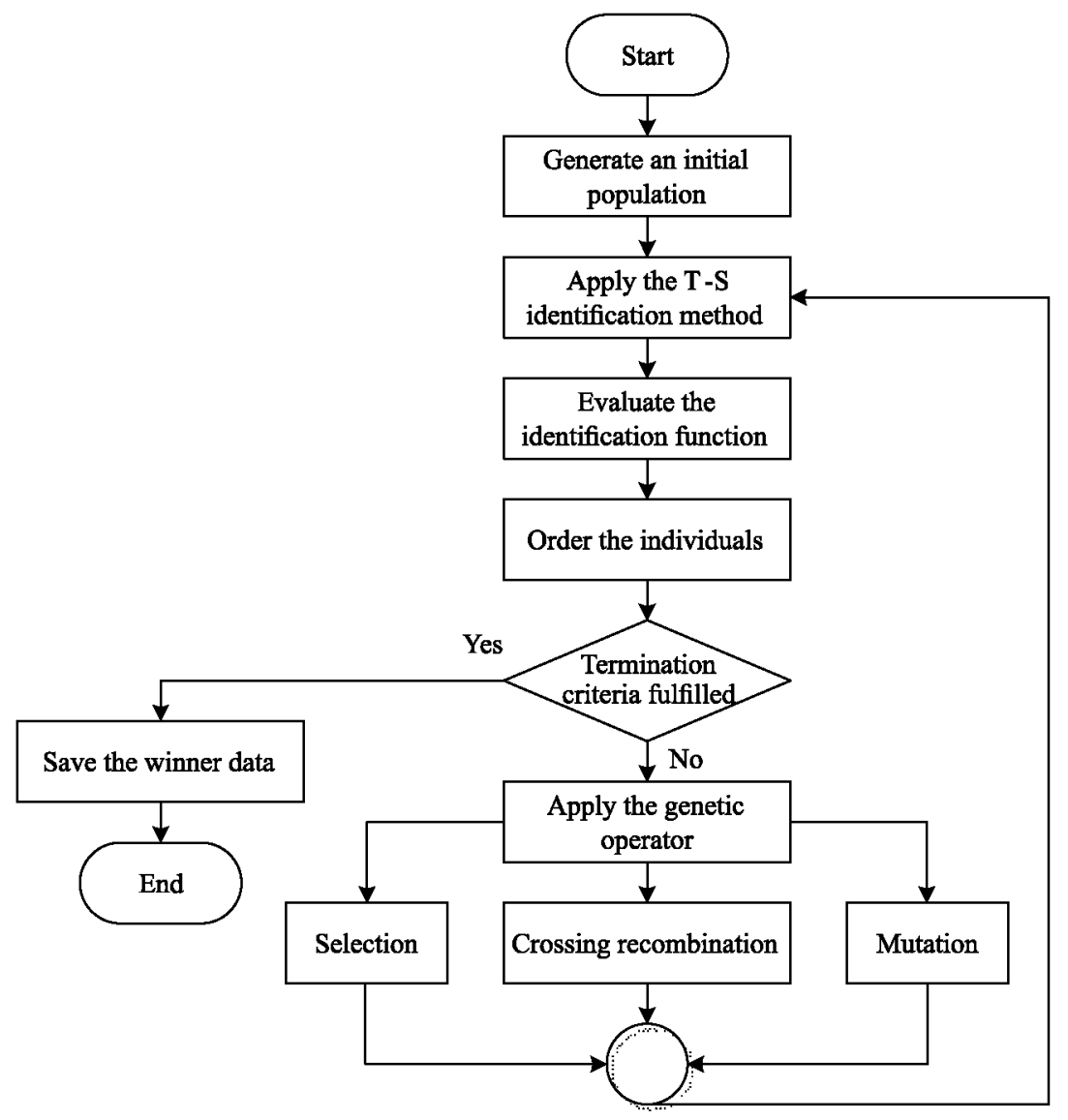

Fig. 2. GA for MDMFs adjustment.

2. For each individual of the population in the current generation, a T-S identification based on MDMFs is performed, obtaining the T-S consequents of each fuzzy rule, as is shown in Section 3.

3. The identification results of each individual are verified with respect to a function $E$, defined as the weighted mean for each output of the Root Mean Squared Error (RMSE) of the identification error.

$E=\sum_{i=1}^{s} \frac{\operatorname{RMSE}\left(y_{i}(k)-\hat{y}_{i}(k)\right)}{s \cdot \operatorname{RMSE}\left(y_{i}(k)\right)}$

The objective of the GA is minimizing the function $E$, thus minimizing the identification error, through the optimal adjustment of the MDMFs parameters $\left(z_{i}\right.$ and $\left.p_{i}\right)$.

4. The population individuals are ordered in the current generation according to their value obtained in the evaluation function $E$, from low to high. The individual which has the lower value in $E$, will become the winner of the population in the current generation.

5. The termination criterion is checked. If the specified maximum number of generations is reached or the value in $E$ obtained by the winner is less than a defined value, then the algorithm ends and the $\mathrm{T}-\mathrm{S}$ antecedents and consequents of the winner are saved. If the termination criterion is not fulfilled, the algorithm continues.

6. The following genetic operators (selection, cross recombination and mutation) are applied on the ordered population of individuals in the current generation to obtain a new population of individuals for the next generation. The genetic operators are only applied to the T-S antecedents.
- Selection: the winner is chosen for the next generation. This operator ensures that an optimal individual could be preserved through generations.

- Cross recombination: some descendants for the next generation are generated by the combination of the best individuals. These operators allow to look for an optimal individual near the best individuals, therefore it allows a small range search but very deep one.

- Mutation: some (or all) characteristics of some individuals are randomly modified for the next generation. These operators are necessary to avoid local minima, since its random search can be made in the full range.

Then, the algorithm continues in step 2, updating the population of individuals for the next generation.

The objective function of the GA is designed to minimize the T$S$ identification error. In other words, the proposed GA is applied to find the best parameters for MDMFs which minimize the T-S identification error.

The proposed method has no restrictions on the GA parameters, operators and their implementation. Thus, without loss of generality, more details about how the proposed method can be applied and the genetic operators can be defined, are presented in Section 5.

A faster convergence of the algorithm has been obtained by taking the centre point $\left(z_{i}\right)$ of the central MDMF of each individual as the gravity centre of the system data, since it is the most representative point. In this way, this centre point of the central MDMF is kept constant throughout the GA generations, facilitating the convergence of the rest of the MDMFs to their optimal locations.

It should be noted that in each individual of the population, a complete fuzzy system has been defined. It is formed by a set 


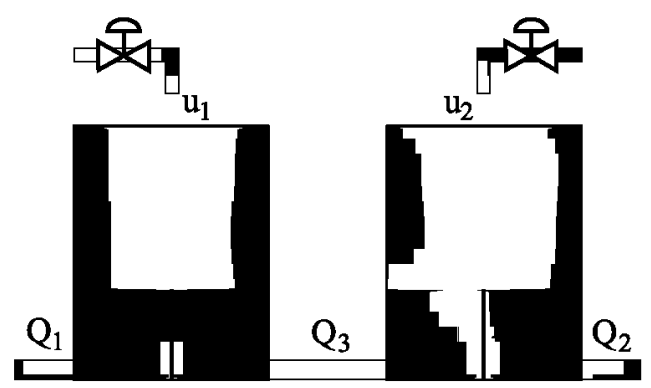

Fig. 3. Coupled tanks system.

of fuzzy rules, which have a set of T-S antecedents (defining the MDMFs) and T-S consequents (modelling the system dynamic behaviour). Thus, the proposed method works with a huge set of parameters, which are treated as follows: the GA adjusts the T-S antecedents of the fuzzy rules, while the T-S identification method obtains the T-S consequents for each fuzzy rule.

Note that, the proposed approach develops an identification of nonlinear multivariable systems in an offline stage, thus the computing time is not a determining factor in the MDMFs parameters adjustment algorithm. On the other hand, reducing the computational cost in the MDMF function (7) is necessary since this function should also be implemented in the fuzzy controller, which is usually an online stage.

\section{Illustrative example}

The presented method is proposed to be applied to any nonlinear system identification based on Takagi-Sugeno fuzzy model. The method could be applied to any nonlinear system with any number of fuzzy variables.

In this section, it is shown through an illustrative example, a coupled tanks system, the advantages of the proposed $\mathrm{T}-\mathrm{S}$ model based on MDMFs adjusted by a GA, in comparison with T-S method based on fuzzy inference of 1DMFs. A coupled tanks system has been chosen since this example fulfils all the advantages and shows a clear graphical representation of the problem and its solution.

\subsection{Coupled tanks system}

A coupled tanks system [38], shown in Fig. 3, consists of two vertical tanks interconnected by a flow pipe, which causes the levels of the two tanks to interact. Each tank has an independent fluid valve for liquid inlet $\left(\mathfrak{u}_{1}, \mathfrak{u}_{2}\right)$ and a liquid outlet flow $\left(Q_{1}, Q_{2}\right)$. Also, there is a flow rate $Q_{3}$ in the pipe connecting the two tanks.

Considering mass balance, the dynamic equation of each tank is developed as follows:

$u_{1}-Q_{1}-Q_{3}=A_{1} \frac{d h_{1}}{d t}$

$u_{2}-Q_{2}+Q_{3}=A_{2} \frac{d h_{2}}{d t}$

where $h_{1}$ and $h_{2}$ are the heights of liquid in each tank. $A_{1}$ and $A_{2}$ are the cross-sectional areas of each tank.

Applying Bernoulli's equation for a non-viscous incompressible fluid in steady flow:

$Q_{1}=\alpha_{1} \sqrt{2 g h_{1}}$

$Q_{2}=\alpha_{2} \sqrt{2 g h_{2}}$

$Q_{3}=\operatorname{sign}\left(h_{1}-h_{2}\right) \alpha_{3} \sqrt{2 g\left|h_{1}-h_{2}\right|}$

where $\alpha_{1}, \alpha_{2}$ and $\alpha_{3}$ are proportionality constants which depend on the coefficients of discharge and the cross-sectional area. The connection flow rate $Q_{3}$ is assumed positive if $h_{1}$ is higher than $h_{2}$.
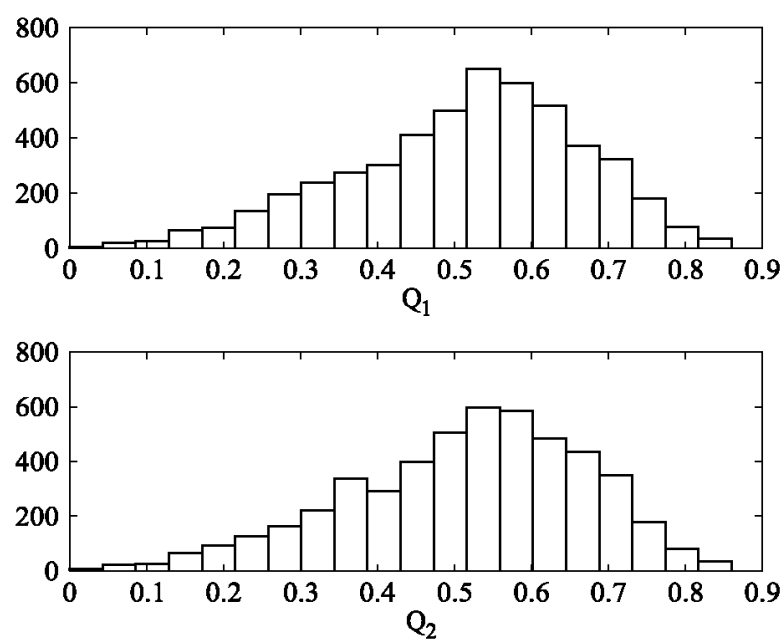

Fig. 4. System data histograms.
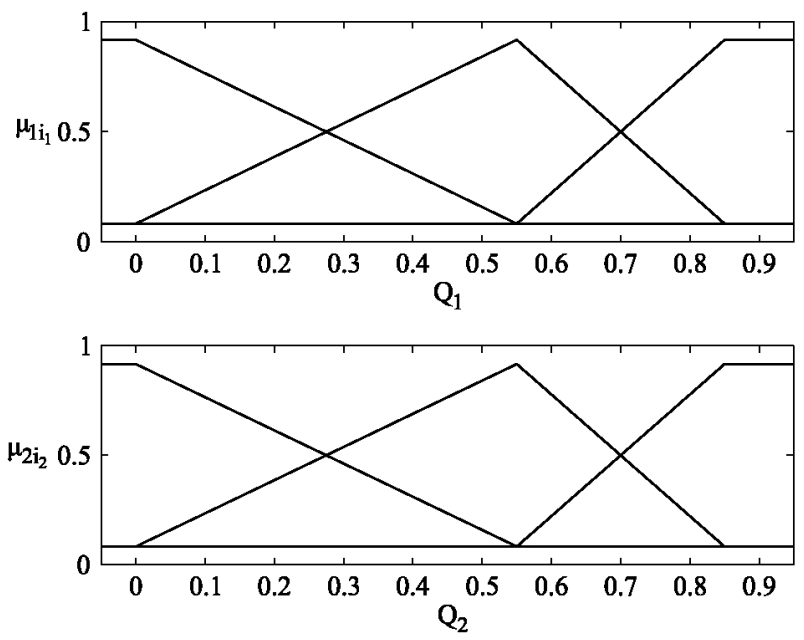

Fig. 5. 1DMFs applied for T-S model.

Supposing the following process values:

$A_{1}=A_{1}=1 \mathrm{~m}^{2}$

$\alpha_{1}=\alpha_{2}=\alpha_{3}=0.1$

$g=9.8 \mathrm{~m} / \mathrm{s}^{2}$

\subsection{T-S identification of a coupled tanks system}

The coupled tanks system can be modelled taking as state variables the output flow rates $Q_{1}$ and $Q_{2}$, with two inputs $u_{1}$ and $u_{2}$. The sampling time for the discrete model is supposed to be $T=1 \mathrm{~s}$.

Fig. 4 shows the system data histograms. Since the fuzzy variables are selected as the state variables themselves $\left(Q_{1}\right.$ and $\left.Q_{2}\right)$, based on the histograms of the system data, it seems reasonable to define the 1DMFs as three triangular functions overlapped by pairs (Fig. 5).

Thus, the fuzzy rules (Fig. 6) for the T-S model [2,7] are obtained by the fuzzy inference of the 1DMFs:

$$
\begin{aligned}
& S^{\left(i_{1}, i_{2}\right)}: \text { If } Q_{1}(k) \text { is } M_{1}^{i_{1}} \text { and } Q_{2}(k) \text { is } M_{2}^{i_{2}} \text { then: } \\
& w^{\left(i_{1}, i_{2}\right)}\left(Q_{1}(k), Q_{2}(k)\right)=\mu_{1 i_{1}}\left(Q_{1}(k)\right) \cdot \mu_{2 i_{2}}\left(Q_{2}(k)\right)
\end{aligned}
$$




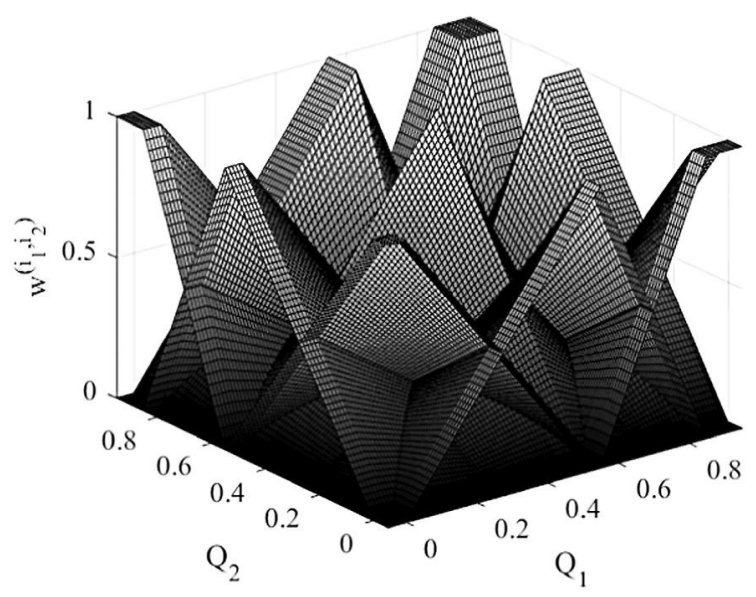

Fig. 6. Fuzzy inference of 1DMFs for T-S model.

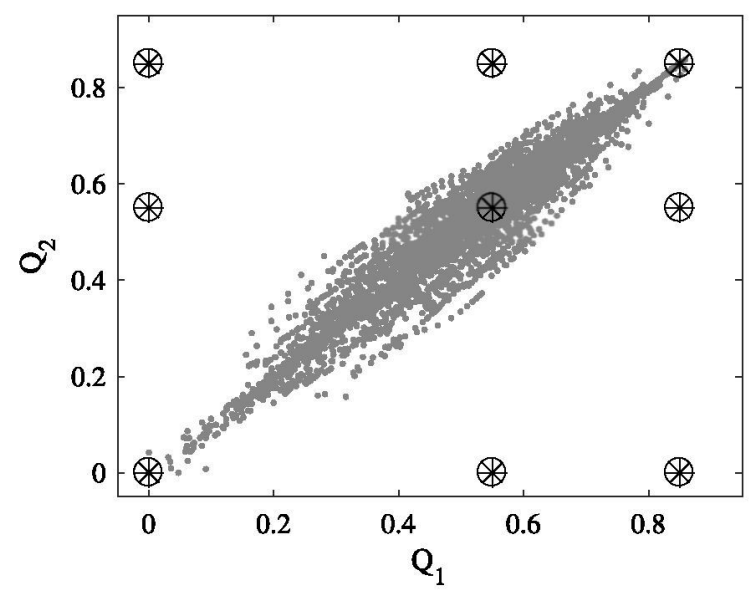

Fig. 7. Fuzzy inference central points projected over the system data space.

Fig. 7 represents the central points of the functions obtained by fuzzy inference, projected over the two-dimensional space of the system data.

The linearized model parameters $P_{0}$ are calculated using the classical least squares method over the system data, with an estimation error of 0.0101 .

$$
\begin{aligned}
{\left[\begin{array}{l}
Q_{1}(k+1) \\
Q_{2}(k+1)
\end{array}\right] } & =\left[\begin{array}{l}
a_{10} \\
a_{20}
\end{array}\right]+\left[\begin{array}{ll}
a_{11} & a_{12} \\
a_{21} & a_{22}
\end{array}\right]\left[\begin{array}{l}
Q_{1}(k) \\
Q_{2}(k)
\end{array}\right] \\
& +\left[\begin{array}{ll}
b_{11} & b_{12} \\
b_{21} & b_{22}
\end{array}\right]\left[\begin{array}{l}
u_{1}(k) \\
u_{2}(k)
\end{array}\right]
\end{aligned}
$$

$P_{0}=\left[\begin{array}{lllll}a_{10} & a_{11} & a_{12} & b_{11} & b_{12} \\ a_{20} & a_{21} & a_{22} & b_{21} & b_{22}\end{array}\right]^{t}$

$$
P_{0}=\left[\begin{array}{lllll}
0.0014 & 0.6066 & 0.2064 & 0.1262 & 0.0585 \\
0.0005 & 0.1918 & 0.6238 & 0.0584 & 0.1246
\end{array}\right]^{t}
$$

With the linearized system parameters $P_{0}$, the 1DMFs shown in Fig. 5 and the weighting factor $\gamma=10^{-6}$, obtained by trial and error, an estimation error of 0.0043 is obtained using the above T$\mathrm{S}$ identification method developed by the authors in $[7,8]$. The T-S model becomes:

$$
\begin{gathered}
\mathrm{S}^{\left(i_{1}, i_{2}\right)} \text { : If } Q_{1}(k) \text { is } M_{1}^{i_{1}} \text { and } Q_{2}(k) \text { is } M_{2}^{i_{2}} \text { then: } \\
{\left[\begin{array}{l}
Q_{1}(k+1) \\
Q_{2}(k+1)
\end{array}\right]=\left[\begin{array}{l}
a_{10}^{\left(i_{1}, i_{2}\right)} \\
a_{20}^{\left(i_{1}, i_{2}\right)}
\end{array}\right]+\left[\begin{array}{ll}
a_{11}^{\left(i_{1}, i_{2}\right)} & a_{12}^{\left(i_{1}, i_{2}\right)} \\
a_{21}^{\left(i_{1}, i_{2}\right)} & a_{22}^{\left(i_{1}, i_{2}\right)}
\end{array}\right]\left[\begin{array}{l}
Q_{1}(k) \\
Q_{2}(k)
\end{array}\right]} \\
+\left[\begin{array}{ll}
b_{11}^{\left(i_{1}, i_{2}\right)} & b_{12}^{\left(i_{1}, i_{2}\right)} \\
b_{21}^{\left(i_{1}, i_{2}\right)} & b_{22}^{\left(i_{1}, i_{2}\right)}
\end{array}\right]\left[\begin{array}{l}
u_{1}(k) \\
u_{2}(k)
\end{array}\right]
\end{gathered}
$$

In Fig. 7, it can be seen how the fuzzy inference of 1DMFs produces fuzzy rules far from the system data, such as those at the top left and bottom right, which have no relevance to the identification algorithm. These fuzzy rules introduce an unnecessary computational cost for the control stage, since the fuzzy controller must consider more fuzzy rules than those which are indeed used.

Note that these fuzzy rules away from the system data (Fig. 7) have been produced by the fuzzy inference process and they have not been generated by a design error, since the 1DMFs (Fig. 5) have been confirmed with the system data histograms (Fig. 4). The generated fuzzy rules by the fuzzy inference process are usually not optimal and sometimes do not even correspond to the system data.

5.3. Modelling and identification of a coupled tanks system applying the proposed method

As it has been described above, the coupled tanks system is modelled by taking the output flow rates $Q_{1}$ and $Q_{2}$ as state variables, with two inputs $u_{1}$ and $u_{2}$. Thus, the proposed T-S model based on MDMFs becomes:

$$
\begin{aligned}
& S^{i} \text { : If } z(k) \text { is } M^{i} \text { then: } \\
& {\left[\begin{array}{l}
Q_{1}(k+1) \\
Q_{2}(k+1)
\end{array}\right]=\left[\begin{array}{l}
a_{10}^{i} \\
a_{20}^{i}
\end{array}\right]+\left[\begin{array}{ll}
a_{11}^{i} & a_{12}^{i} \\
a_{21}^{i} & a_{22}^{i}
\end{array}\right]\left[\begin{array}{l}
Q_{1}(k) \\
Q_{2}(k)
\end{array}\right]} \\
& +\left[\begin{array}{ll}
b_{11}^{i} & b_{12}^{i} \\
b_{21}^{i} & b_{22}^{i}
\end{array}\right]\left[\begin{array}{l}
u_{1}(k) \\
u_{2}(k)
\end{array}\right]
\end{aligned}
$$

where the fuzzy variables are the state variables themselves:

$z=\left[\begin{array}{ll}Q_{1} & Q_{2}\end{array}\right]^{t}$

Five fuzzy rules are used to define the T-S fuzzy system. The number of fuzzy rules have been defined through a trial and error adjustment over the proposed example. Many trials have been made. It has been found that five fuzzy rules offer a good compromise between computational cost and identification error.

In the GA, the population is formed by fifteen individuals. The GA ends when one hundred generations are reached. In order to obtain the next generation of individuals, the applied genetic operators, in the ordered population of individuals, are:

- For individual 1: Selection. The winner is updated for the next generation without changes.

- For individuals 2, 3, 4, 5: Cross recombination. These individuals are combined with the winner for the next generation.

- Individuals 2 and 3: The parameters are obtained from the winner, but the parameters of one of the rules are obtained taking the mean of these parameters from the winner and individuals 2 and 3 respectively.

- Individuals 4 and 5: The parameters are obtained from individuals 2 and 3 respectively, but the parameters of one of the rules are obtained taking the mean of these parameters from the winner and individuals 2 and 3 respectively.

- For individuals 6, 7, 8, 9, 10, 11: Small mutation. Small random changes are applied over individuals 1 and 2 . 


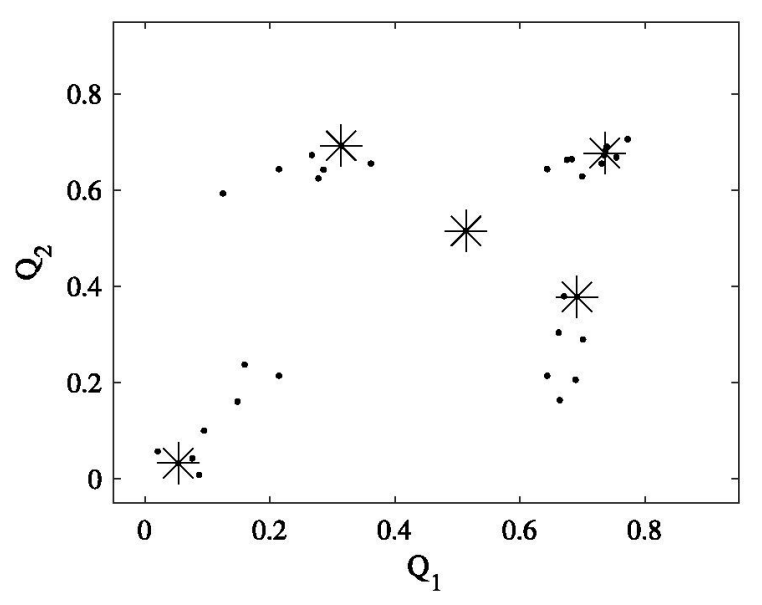

Fig. 8. MDMFs centres of the winner at each iteration.

- Individual 6: The parameters are obtained from the winner, but random increments over the parameters of one of the rules are added.

- Individual 7: The parameters are obtained from the winner, but a random increment over the position parameter $z_{i}$ of one of the rules is added.

- Individual 8: The parameters are obtained from the winner, but a random increment over the distance weighting parameter $p_{i}$ of one of the rules is added.

- Individual 9: The parameters are obtained from individual 2, but random increments over the parameters of one of the rules are added.

- Individual 10: The parameters are obtained from individual 2 , but a random increment over the position parameter $z_{i}$ of one of the rules is added.

- Individual 11: The parameters are obtained from individual 2 , but a random increment over the distance weighting parameter $p_{i}$ of one of the rules is added.

- For individuals 12 and 13: Large mutation. Large random changes are applied over the winner. The parameters are obtained from the winner, but the parameters of one of the rules are randomly chosen.

- For individuals 14 and 15: Complete mutation. New random individuals are generated for the next generation.

In the complete algorithm, there are fifteen individuals in the GA population. Each one with five fuzzy rules. Each fuzzy rule has as T-S antecedents the MDMF parameters, the centre point $z_{i} \in \mathfrak{R}^{2}$ and the distance weighting $p_{i} \in \mathfrak{R}^{2}$. Moreover, each fuzzy rule has as $\mathrm{T}-\mathrm{S}$ consequents, ten parameters of the T-S fuzzy state model. Thus, the algorithm works with 1050 parameters (15 5 . $(2 \cdot 2+10))$. Thus, the proposed algorithm works as follows:

- The GA manages the T-S antecedents of each individual for all fuzzy rules (20 parameters $(5 \cdot 2 \cdot 2)$ per individual).

- With the defined fuzzy rules, the T-S consequents of each individual are obtained by T-S identification method [2] (50 parameters (5 10 ) per individual).

Applying the GA for MDMFs design with the above described characteristics, the following results are obtained:

Fig. 8 shows the MDMFs centre points of the winner at each iteration. Note that the centre point $\left(z_{i}\right)$ of the central MDMF remains static throughout the GA generations, because it is defined as the centre of gravity of the system data.

Fig. 9 shows the centre points of the MDMFs obtained at the end of the GA algorithm over the system data.

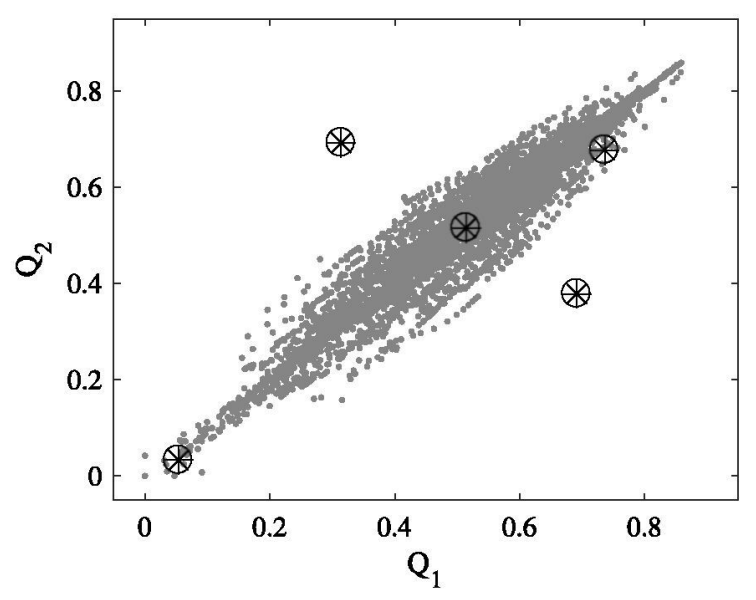

Fig. 9. System data and obtained MDMFs centres.

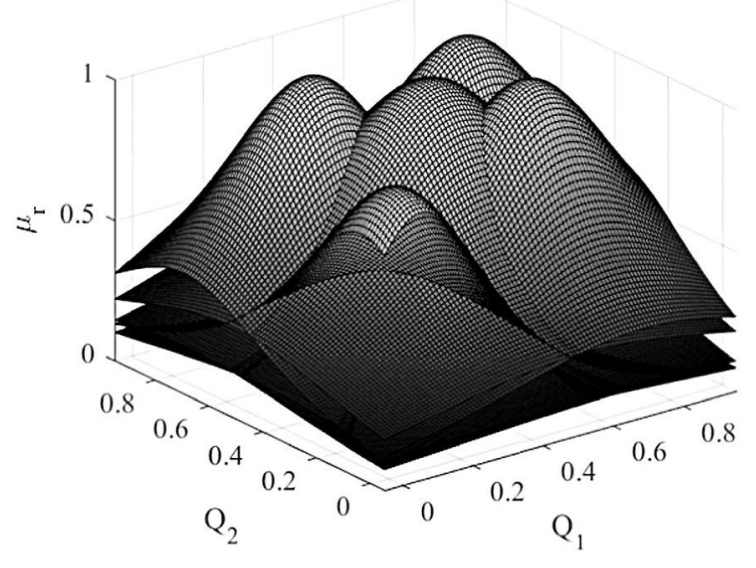

Fig. 10. MDMFs obtained with the proposed algorithm.

In Fig. 10, the final MDMFs obtained for the fuzzy system with the proposed algorithm are shown.

Since the GA has an important random component, one hundred tests have been performed and the results of the estimation error of T-S identification using MDMFs, throughout the GA generations, are shown in Fig. 11. The mean of the T-S identification error during the GA generations is represented in continuous line. Maximums and minimums are drawn in dashed line. Percentiles 10 and 90 are shown in dash-dot line. The T-S identification error through the GA generations obtained in this work shown test is represented in thick continuous line.

It can be seen in Fig. 9, that the fuzzy rules are not restricted to 1DMFs elements combination, such as using the fuzzy inference method (Fig. 7). The use of MDMFs allows that the fuzzy rules can be placed at any point of the multi-dimensional space. Thus, a better adjustment over the system data can be obtained, improving the identification results through a smaller number of fuzzy rules.

An estimated error of 0.0035 is obtained. In addition, the results obtained with the T-S identification $[7,8]$ have been performed with 9 fuzzy rules, whereas in the proposed identification method only 5 multidimensional fuzzy rules have been used. Thus, not only better results are obtained in the identification, but it has been reduced the number of fuzzy rules.

It can be seen in Fig. 11 how the identification error decreases throughout the GA generations. All the tests give similar results, obtaining better results than the T-S identification method $[7,8]$ with a smaller number of fuzzy rules. From this one hundred tests, 


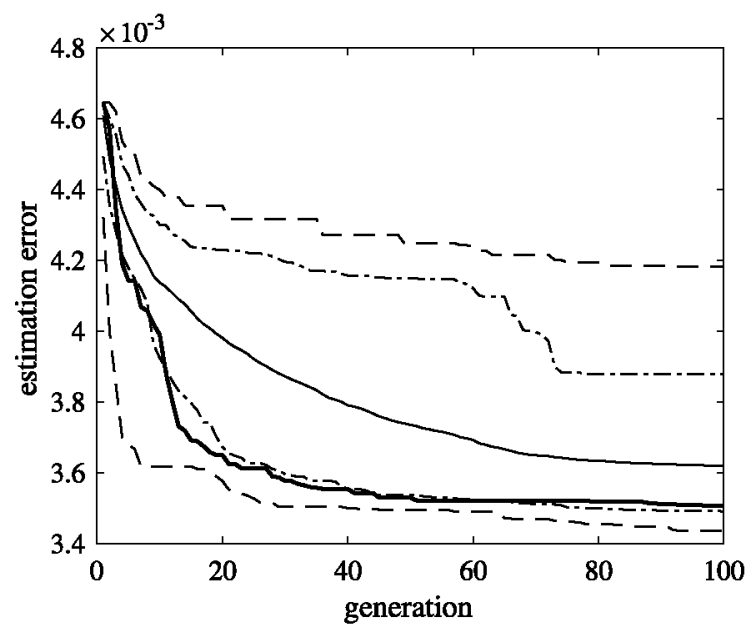

Fig. 11. Estimation error of T-S identification using MDMFs throughout the GA generations. (a) Continuous line: mean of 100 tests. (b) Dashed line: maximum and minimum of 100 tests. (c) dash-dot line: percentiles 10 and 90 of 100 hundred tests. (d) Thick continuous line: test shown in the article.

Table 1

Probability of occurrence of the genetics operators.

\begin{tabular}{cl}
\hline Individual & Occurrence (\%) \\
\hline 1 & 61.95 \\
2 & 6.09 \\
3 & 5.94 \\
4 & 1.72 \\
5 & 0.97 \\
6 & 2.75 \\
7 & 2.45 \\
8 & 7.70 \\
9 & 1.73 \\
10 & 1.61 \\
11 & 5.24 \\
12 & 0.88 \\
14 & 0.87 \\
15 & 0.03 \\
\hline
\end{tabular}

the occurrence probability of the genetic operators applied over the individuals previously defined is shown in Table 1.

\section{Conclusions}

In this paper, a new method for the $\mathrm{T}-\mathrm{S}$ model identification of nonlinear multivariable systems using MDMFs is developed. The fuzzy inference method of $1 D M F s$ can produce fuzzy rules that do not correspond to system data. This in turn produces a loss of optimality and an unnecessary computational cost. MDMFs are proposed for solving this problem.

The proposed algorithm for the T-S identification using MDMFs consists in the adjustment of T-S antecedents (the MDMFs parameters) using a GA and the calculation of the T-S consequents using the T-S identification method.

An illustrative example shows that the proposed method provides a fewer identification error with a smaller number of fuzzy rules than the 1DMFs fuzzy inference method.

\section{Acknowledgements}

This work is funded by Spanish Ministry of Economy and Competitiveness (Assisted Navigation through Natural Language (NAVEGASE) project (DPI2014-53525-C3-1-R)). 